\title{
Cardiovascular complications after common bile duct stone extractions
}

\author{
Eva-Lena Syrén $^{1,2}\left[\right.$ [ Lars Enochsson ${ }^{3} \cdot$ Staffan Eriksson $^{1,2} \cdot$ Arne Eklund $^{1,2} \cdot$ Bengt Isaksson $^{1} \cdot$ Gabriel Sandblom $^{4,5}$
}

Received: 31 March 2020 / Accepted: 25 June 2020 / Published online: 1 July 2020

(c) The Author(s) 2020

\begin{abstract}
Background Common bile duct stone (CBDS) is a common condition the rate of which increases with age. Decision to treat in particular elderly and frail patients with CBDS is often complex and requires careful assessment of the risk for treatmentrelated cardiovascular complications. The aim of this study was to compare the rate of postoperative cardiovascular events in CBDS patients treated with the following: ERCP only; cholecystectomy only; cholecystectomy followed by delayed ERCP; cholecystectomy together with ERCP; or ERCP followed by delayed cholecystectomy.

Methods The study was based on data from procedures for gallstone disease registered in the Swedish National Quality Register for Cholecystectomy and Endoscopic Retrograde Cholangiopancreatography (GallRiks) 2006-2014. ERCP and cholecystectomy procedures performed for confirmed or suspected CBDS were included. Postoperative events were registered by cross-matching GallRiks with the National Patient Register (NPR). A postoperative cardiovascular event was defined as an ICD-code in the discharge notes indicating myocardial infarct, pulmonary embolism or cerebrovascular disease within 30 days after surgery. In cases where a patient had undergone ERCP and cholecystectomy on separate occasions, the 30-day interval was timed from the first intervention.

Results A total of 23,591 underwent ERCP or cholecystectomy for CBDS during the study period. A postoperative cardiovascular event was registered in 164 patients and death within 30 days in 225 patients. In univariable analysis, adverse cardiovascular event and death within 30 days were more frequent in patients who underwent primary $\operatorname{ERCP}(p<0.05)$. In multivariable analysis, adjusting for history of cardiovascular disease or events, neither risk for cardiovascular complication nor death within 30 days remained statistically significant in the ERCP group.

Conclusions Primary ERCP as well as cholecystectomy may be performed for CBDS with acceptable safety. More studies are required to provide reliable guidelines for the management of CBDS.
\end{abstract}

Keywords ERCP · Choledocholithiasis · Cardiovascular complication

Common bile duct stone (CBDS) is a common disease with varying clinical manifestations. CBD stones are often

Eva-Lena Syrén

eva.lena.syren@akademiska.se

1 Department of Surgical Sciences, Uppsala University, 75135 Uppsala, Sweden

2 Centre for Clinical Research, Västmanland Hospital, Västerås, Sweden

3 Department of Surgical and Perioperative Sciences, Sunderby Research Unit, Umeå University, SurgeryUmeå, Sweden

4 Department of Clinical Science and Education Södersjukhuset, Karolinska Institute, Stockholm, Sweden

5 Department of Surgery, Södersjukhuset, Stockholm, Sweden asymptomatic, but may cause biliary pancreatitis, obstructive jaundice, cholangitis, or recurrent pain $[1,2]$. There are several accepted methods of treatment for CBDS [3-8]. Cholecystectomy with or without concomitant intraoperative rendezvous endoscopic retrograde cholangiopancreaticography (ERCP), if CBDS is found on intraoperative cholangiography (IOC), is a well-established, safe and cost-effective method for patients considered fit for surgery [9-12].

ERCP is sometimes performed as part of a two-stage procedure, either as ERCP followed by delayed cholecystectomy or cholecystectomy followed by delayed ERCP $[1,13]$. In some patients, where high age and comorbidity render them too high risk for surgery, ERCP with sphincterotomy and stone extraction may be preferred as sole intervention, even if recurrent choledocholithiasis is more common 
when ERCP is the only treatment performed [14]. If ERCP is performed without the aid of antegrade introduction of a guidewire at IOC, $4-18 \%$ of attempts fail due to inability to cannulate the bile duct [9]. Surgical complications, especially post-ERCP pancreatitis (PEP), are also more frequent after standard ERCP compared to rendezvous ERCP [10, 11, 15-17].

The frequency of CBDS increases with age. This complicates management as comorbidity and frailty increase the risk for intervention-related complications. Cardiovascular disease and biliary stone disease share risk factors such as obesity, hypertension, diabetes, dyslipidemia, and cigarette smoking [18-20]. There also appears to be an association between gallstone disease and cardiovascular disease [21].

The cardiovascular complication and pulmonary thromboembolism (PTE) rates following laparoscopic cholecystectomy and ERCP are low, even in elderly patients $(<2 \%)$ [22-25].

The aim of this study was to compare postoperative cardiovascular complication rates (myocardial infarct, pulmonary thromboembolism and/or cerebrovascular disease) in patients with CBDS treated with: ERCP only; cholecystectomy only; cholecystectomy followed by delayed ERCP; cholecystectomy combined with ERCP; or ERCP followed by delayed cholecystectomy.

\section{Materials and methods}

This study was based on procedures for gallstone disease registered in the Swedish National Quality Register for Cholecystectomy and Endoscopic Retrograde Cholangiopancreatography (GallRiks) 2006-2014. GallRiks registration began 1st May 2005 and now covers approximately $90 \%$ of all cholecystectomies and ERCPs performed in Sweden, including patient- and procedure-related data. All intra- and postoperative adverse events, including cardiovascular complications, are registered, and the completeness of 30-day follow-up of postoperative complications is approximately $95 \%$. GallRiks is regularly externally validated $[26,27]$.

In the present study, ERCP as well as cholecystectomy performed with confirmed or suspected CBDS as indication were included. Patients who underwent cholecystectomy combined with ERCP were also included as long as either of the procedures was performed with CBDS as indication. ERCP or cholecystectomy performed because of malignant stricture or suspicion of cancer were excluded as well as patients who underwent one or more procedures without CBDS as indication.

Patients with confirmed or suspected CBDS were divided into five treatment groups: ERCP only; cholecystectomy only; cholecystectomy followed by delayed ERCP, cholecystectomy combined with ERCP; or ERCP followed by delayed cholecystectomy.

Postoperative events were registered by cross-matching GallRiks with the National Patient Register (NPR). Data on cardiovascular complications within 30 days after surgery, defined as a diagnosis in the discharge notes with an ICDcode indicating myocardial infarct, pulmonary embolism or cerebrovascular disease (not including those who had an ICD-code indicating cerebrovascular disease prior to surgery), were retrieved from the NPR. If a patient had undergone both ERCP and cholecystectomy, the 30-day interval was timed from the first intervention. Data on previous cardiovascular events were also obtained from the NPR.

The Regional Ethics Review Board in Stockholm approved the study 18th March 2015 (IRB-approval, reference number: 2015/339-31/1).

Consent from the patient to participate in register-based research is required for registration in GallRiks. Patients are given the opportunity to withdraw all their personal data at any time from the register.

\section{Statistics}

In order to adjust for confounders, multivariate logistic regression analyses were performed, with cardiovascular event (myocardial infarct and/or pulmonary embolus and/or cerebrovascular disease) and death within 30 days as endpoints. The multivariate models were based on age ( $\geq 80$ years vs $<80$ years), ASA score (III-V vs I-II), gender, treatment and history of cardiovascular condition or event (myocardial infarct, heart failure, peripheral vascular disease, cerebrovascular event, diabetes with secondary complication or pulmonary embolism). Patients who underwent cholecystectomy and ERCP during the same procedure and those who underwent cholecystectomy and delayed ERCP were grouped together with the cholecystectomy group, whereas those who underwent ERCP and delayed cholecystectomy were grouped together with the ERCP group. This grouping was based on which procedure was the primary intervention aimed at managing the CBDS.

Poisson regression was used to calculate the 30-day ageand gender-adjusted standardized mortality ratio (SMR) based on the expected mortality rate extrapolated from the Swedish general population in 2007.

\section{Results}

During the study period, 103,208 patients underwent cholecystectomy and/or ERCP due to gallstone disease. After excluding cholecystectomies performed without preoperatively diagnosed common bile duct stone and 
patients registered with more than one cholecystectomy, 23,591 patients remained in the study group. Of those, 8790 underwent ERCP only, 10,362 cholecystectomy only, 1032 cholecystectomy followed by delayed ERCP, 1258 cholecystectomy combined with ERCP, and 2149 ERCP followed by delayed cholecystectomy (Fig. 1).
Patients in the ERCP only group were older, more often female and ASA grade III-V vs I-I compared to the cholecystectomy only group. A previous history of cardiovascular disease (myocardial infarct, heart failure, peripheral vascular disease, cerebrovascular event, diabetes with secondary complication or pulmonary embolism) was also much more common in the ERCP only group. In the group ERCP

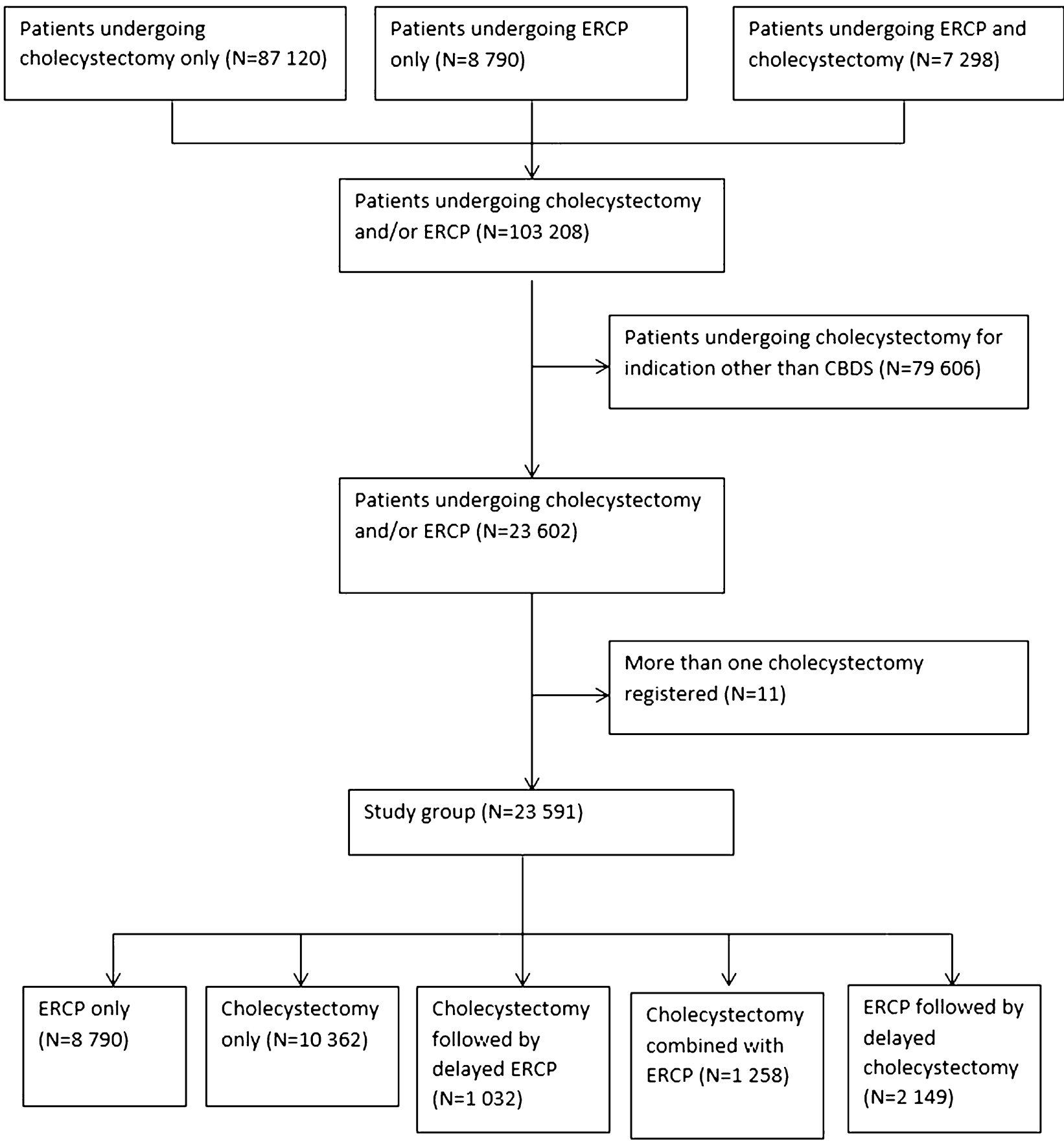

Fig. 1 Flow chart. Confirmed or suspected CBDS as indication for treatment 
followed by delayed cholecystectomy, patients were older and more often had a previous history of cardiovascular disease compared to patients in the groups cholecystectomy combined with ERCP, and cholecystectomy followed by delayed ERCP (Table 1).

In all, a postoperative cardiovascular event was registered in 164 cases and death within 30 days in 225 cases. Postoperative adverse event and death within 30 days were more frequently seen in the ERCP only group compared to the other groups. Myocardial infarct was at least twice as common $(0.71 \%)$ and cerebrovascular lesion at least three times as common $(0.26 \%)$ in the ERCP only group compared to the other groups. The incidence of pulmonary embolism was more equally distributed between groups and most common in the group cholecystectomy followed by delayed ERCP $(0.39 \%)$. Postoperative death within 30 days was between 5 and 20 times more common in the ERCP only group (1.97\%) (Table 2).

Age $\geq 80$ years, ASA $>1$ and history of cardiovascular disease or event were all risk factors for postoperative complication and death. In the univariable and multivariable logistic regression analyses, cardiovascular complication and death within 30 days were studied in the ERCP group (ERCP only + ERCP with delayed cholecystectomy), with

Table 1 Baseline characteristics

\begin{tabular}{|c|c|c|c|c|c|}
\hline & ERCP only $(N=8790)$ & $\begin{array}{l}\text { Cholecystectomy } \\
\text { only }(N=10,362)\end{array}$ & $\begin{array}{l}\text { Cholecystectomy } \\
\text { followed by delayed } \\
\operatorname{ERCP}(N=1032)\end{array}$ & $\begin{array}{l}\text { Cholecystectomy } \\
\text { combined with ERCP } \\
(N=1258)\end{array}$ & $\begin{array}{l}\text { ERCP followed by } \\
\text { delayed cholecystectomy } \\
(N=2149)\end{array}$ \\
\hline \multicolumn{6}{|l|}{ Gender } \\
\hline Men & $3653(36.1 \%)$ & $4650(46.0 \%)$ & $413(4.1 \%)$ & $479(4.7 \%)$ & $918(9.1 \%)$ \\
\hline Women & $5137(38.1 \%)$ & $5712(42.4 \%)$ & $619(4.6 \%)$ & $779(5.8 \%)$ & $1231(9.1 \%)$ \\
\hline $\begin{array}{l}\text { Mean age, years } \\
\text { (standard deviation) }\end{array}$ & $73.5(15.5)$ & $53.5(17.8)$ & $55.0(17.9)$ & $49.4(18.4)$ & $58.8(16.1)$ \\
\hline \multicolumn{6}{|l|}{$A S A$} \\
\hline I & $1583(18.9 \%)$ & $4631(56.1 \%)$ & $429(5.2 \%)$ & $646(7.8 \%)$ & $980(11.9 \%)$ \\
\hline II & $4627(41.5 \%)$ & $4570(41.0 \%)$ & $468(4.2 \%)$ & $515(4.6 \%)$ & $985(8.7 \%)$ \\
\hline III & $2451(61.9 \%)$ & $1092(27.8 \%)$ & $128(3.2 \%)$ & $95(2.4 \%)$ & $191(4.8 \%)$ \\
\hline IV & $148(62.7 \%)$ & $66(28.0 \%)$ & $7(3.0 \%)$ & $2(0.8 \%)$ & $13(5.5 \%)$ \\
\hline V & $1(25.0 \%)$ & $3(75.0 \%)$ & 0 & 0 & 0 \\
\hline \multicolumn{6}{|c|}{ History of cardiovascular disease and events } \\
\hline Myocardial infarct & $1140(13.0 \%)$ & $373(3.6 \%)$ & $49(4.7 \%)$ & $35(2.8 \%)$ & $103(4.8 \%)$ \\
\hline Cardiac failure & $1406(16.0 \%)$ & $363(3.5 \%)$ & $43(4.2 \%)$ & $34(2.7 \%)$ & $100(4.7 \%)$ \\
\hline $\begin{array}{l}\text { Peripheral vascular } \\
\text { disease }\end{array}$ & $691(7.9 \%)$ & $209(2.0 \%)$ & $19(1.8 \%)$ & $26(2.1 \%)$ & $72(3.4 \%)$ \\
\hline Cerebrovascular event & $1536(17.5 \%)$ & $512(4.9 \%)$ & $54(5.2 \%)$ & $54(4.3 \%)$ & $130(6.0 \%)$ \\
\hline $\begin{array}{l}\text { Diabetes with second- } \\
\text { ary complication }\end{array}$ & $454(5.2 \%)$ & $188(1.8 \%)$ & $21(2.0 \%)$ & $14(1.1 \%)$ & $53(2.5 \%)$ \\
\hline Pulmonary embolism & $270(3.1 \%)$ & $112(1.1 \%)$ & $16(1.6 \%)$ & $7(0.6 \%)$ & $26(1.2 \%)$ \\
\hline
\end{tabular}

Table 2 Postoperative adverse events in confirmed or suspected CBDS within 30 days in the Swedish National Quality Register for Cholecystectomy and Endoscopic Retrograde Cholangiopancreatography (GallRiks) 2006-2014

\begin{tabular}{lllllll}
\hline & $\begin{array}{l}\text { ERCP only } \\
(N=8790)\end{array}$ & $\begin{array}{l}\text { Cholecystectomy } \\
\text { only }(N=10,362)\end{array}$ & $\begin{array}{l}\text { Cholecystectomy } \\
\text { followed by } \\
\text { delayed ERCP } \\
(N=1032)\end{array}$ & $\begin{array}{l}\text { Cholecystectomy } \\
\text { combined with } \\
\text { ERCP }(N=1258)\end{array}$ & $\begin{array}{l}\text { ERCP followed by } \\
\text { delayed cholecys- }\end{array}$ & $\begin{array}{l}\text { Total complication } \\
\text { incidence }(N=164) \\
\text { tectomy }(N=2149) \\
\text { and death }(N=225)\end{array}$ \\
\hline $\begin{array}{l}\text { Myocardial infarct } \\
\text { Cerebrovascular } \\
\quad \text { lesion }\end{array}$ & $23(0.26 \%)$ & $5(0.05 \%)$ & $1(0.10 \%)$ & $0(0 \%)$ & $3(0.14 \%)$ & $2(0.09 \%)$ \\
$\begin{array}{l}\text { Pulmonary embo- } \\
\text { lism }\end{array}$ & $23(0.26 \%)$ & $19(0.18 \%)$ & $4(0.39 \%)$ & $3(0.24 \%)$ & $2(0.09 \%)$ & 31 \\
$\begin{array}{l}\text { Postoperative } \\
\text { death }\end{array}$ & $173(1.97 \%)$ & $43(0.41 \%)$ & $4(0.39 \%)$ & $3(0.24 \%)$ & $2(0.09 \%)$ & 225 \\
\hline
\end{tabular}


the cholecystectomy group as reference (cholecystectomy with or without combined ERCP + cholecystectomy with delayed ERCP). In the univariable analysis, adverse cardiovascular event (OR 2.74, 95\% confidence interval [CI] $1.95-3.84, p<0.001)$ and death (OR 4.10, CI 3.00-5.62, $p<0.001)$ were more frequent in the ERCP group. In the multivariable analysis, adjusting for history of cardiovascular conditions or events, neither the risk for cardiovascular complication (OR 1.12, CI 0.77-1.64, $p<0.548$ ) nor death within 30 days (OR 1.38, CI 0.97-1.96, $p<0.071$ ) remained statistically significant in the ERCP group (Table 3 ).

\section{Discussion}

In this register-based study, we analyzed postoperative cardiovascular complications in a large number of patients who underwent surgical treatment for confirmed or suspected CBDS. The study was based on prospectively assembled population-based data from GallRiks covering a long period of time. The study focused on the most common postoperative cardiovascular events i.e. myocardial infarct and/or pulmonary embolus and/or cerebrovascular disease as well as death within 30 days. Although the study could not show any approach to be safer than the others, our results may help in future treatment-decisions.

We decided to focus on and present only the incidence of cardiovascular complications. There are differences in the burden of cardiovascular disease between Sweden and other parts of the world. U.S. and Swedish data diverge to a lesser extent than what may be seen when the Western World is compared to areas outside Western Europe and North America [28].

The prevalence of gallstone-related symptoms, including CBDS, in the population is high (7-15\%) and high age is a significant risk factor for prolonged hospital stay and death after any procedure for gallstone removal $[29,30]$. The comorbidity rate in elderly patients undergoing treatment for choledocholithiasis is high compared to younger patients [31]. Frailty is a crucial risk factor, although it is difficult to quantify. We consider age as surrogate measure for frailty, although age and frailty only partly correlate.

Tobacco use and obesity are major risk factors that have to be taken into account when estimating the risk for cardiovascular complications following a surgical or endoscopic intervention. Even if smoking and BMI are included in the ASA physical status they were not registered routinely in GallRiks during the period of the study [32]. We also lack data on medications, including anticoagulation. There was no consistent predetermined national algorithm administration during the period of study. In Sweden the prevailing routine is to interrupt anticoagulation therapy before surgery and ERCP and restart anticoagulation postoperatively, but each hospital follow their own local guidelines.

Anesthesia was not included as predictor in the present study. It has though been explored in a recent study based on GallRiks data which has shown more post-procedural complications occurred after ERCPs performed under deep

Table 3 Univariable and multivariable analyses of factors predicting cardiovascular event and death within 30 days after surgical and/or

ish National Quality Register for Cholecystectomy and Endoscopic Retrograde Cholangiopancreatography (GallRiks) 2006-2014 endoscopic treatment for confirmed or suspected CBDS in the Swed-

\begin{tabular}{|c|c|c|c|c|}
\hline \multirow[t]{2}{*}{ Univariable } & \multicolumn{2}{|l|}{ Cardiovascular complication } & \multicolumn{2}{|l|}{ Death } \\
\hline & $\begin{array}{l}\text { Odds ratio (95\% confidence } \\
\text { interval) }\end{array}$ & $p$ & $\begin{array}{l}\text { Odds ratio ( } 95 \% \text { confidence } \\
\text { interval) }\end{array}$ & $p$ \\
\hline Age $\geq 80$ years $($ ref $<80$ years $)$ & $4.37(3.20-5.60)$ & $<0.001$ & $9.60(7.20-12.79)$ & $<0.001$ \\
\hline Men (ref women) & $1.16(0.85-1.59)$ & 0.340 & $1.19(0.91-1.55)$ & 0.197 \\
\hline \multicolumn{5}{|l|}{ ASA I (ref) } \\
\hline ASA II & $3.83(2.16-6.79)$ & $<0.001$ & $6.42(3.08-13.35)$ & $<0.001$ \\
\hline ASA III & $9.82(5.51-17.52)$ & $<0.001$ & $31.39(15.32-64.31)$ & $<0.001$ \\
\hline ASA IV & $26.03(11.44-59.22)$ & $<0.001$ & $150.02(67.94-331.23)$ & $<0.001$ \\
\hline ASA V & - & - & $343.38(32.20-3662.14)$ & $<0.001$ \\
\hline History of cardiovascular disease or event $\mathrm{a}^{\mathrm{a}}$ & $10.20(7.12-14.60)$ & $<0.001$ & $6.25(4.74-8.23)$ & $<0.001$ \\
\hline ERCP (ref cholecystectomy) ${ }^{\mathrm{b}}$ & $2.74(1.95-3.84)$ & $<0.001$ & $4.10(3.00-5.62)$ & $<0.001$ \\
\hline Multivariable & \multicolumn{2}{|l|}{ Cardiovascular complication } & \multicolumn{2}{|l|}{ Death } \\
\hline ERCP (ref cholecystectomy) ${ }^{\mathrm{a}}$ & $1.12(0.77-1.64)$ & 0.548 & $1.38(0.97-1.96)$ & 0.071 \\
\hline
\end{tabular}

${ }^{a}$ History of myocardial infarct, heart failure, peripheral vascular disease, cerebrovascular event, diabetes with secondary complication or pulmonary embolism

${ }^{\mathrm{b}}$ In cases where ERCP as well as cholecystectomy were performed, allocation was determined by the primary procedure. If cholecystectomy and ERCP were performed as one procedure, the procedure was allocated to the cholecystectomy group 
sedation compared to those performed under general anesthesia [33].

The five treatment groups in this study are not predetermined and the affiliation to a certain group is dependent on several heterogeneous factors such as complexity and status of the biliary disease and preference of the deciding doctor or local treatment regimes. Several strategies are employed to manage CBDS disease, and methods and timing vary from hospital to hospital [3]. Even if cholecystectomy combined with rendezvous ERCP is standard in many departments, the decision on which treatment is used for CBDS is usually based on local tradition and ERCP-competence. Before the introduction of intraoperative rendezvous ERCP, it was common that patients with CBDS were treated with a twostage procedure, either preoperative ERCP followed by cholecystectomy or cholecystectomy followed by postoperative ERCP [34]. There are still units where cholecystectomy is performed on regular basis but where there is a lack of ERCP resources and a two-stage procedure thus remains the only choice [34].

Even if early cholecystectomy appears to be safe in elderly, there is a tendency to choose minimally invasive treatment methods such as ERCP when it comes to older, frail patients with comorbidity [35]. No subsequent cholecystectomy was registered for any of the 8790 patients with ERCP as sole intervention. It is, however, possible that some of the patients underwent cholecystectomy after the period of the study. As a cholecystectomy at that late state could not be expected to be performed with the aim of preventing CBDS, we do not think that they are relevant for the aims of the present study.

It is possible that procedure-related complications preceded the cardiovascular complications, which also has to be taken into account when deciding on treatment of common bile duct stones. Even if we believe that most complications are included, it can't be excluded that registration of some adverse events could have been missed in the analysis regarding those patients who underwent both ERCP and cholecystectomy as two separate interventions and with a long interval between procedures.

In this study patients who were selected for ERCP were older and had more comorbidity than patients in the other treatment groups. Myocardial infarction, cardiac failure, peripheral vascular disease, cerebrovascular event, diabetes with secondary complication and pulmonary embolism were strong predictors for cardiovascular complication and death after surgical treatment for CBDS. We believe that the selection of frail patients and patients with greater comorbidity for ERCP explains why ERCP was significant in univariate analysis. In multivariable analysis, adjusting for history of cardiovascular disease or events, neither risk for cardiovascular complication nor death within 30 days remained statistically significant in the ERCP group.
Based on the results of this study we believe both ERCP as well as cholecystectomy may be used for CBDS treatment in the elderly with acceptable safety.

Acknowledgements Open access funding provided by Uppsala University.

\section{Compliance with ethical standards}

Disclosures Drs. Syrén, Enochsson, Eriksson, Eklund, Isaksson and Sandblom have no conflicts of interest or financial ties to disclose.

Open Access This article is licensed under a Creative Commons Attribution 4.0 International License, which permits use, sharing, adaptation, distribution and reproduction in any medium or format, as long as you give appropriate credit to the original author(s) and the source, provide a link to the Creative Commons licence, and indicate if changes were made. The images or other third party material in this article are included in the article's Creative Commons licence, unless indicated otherwise in a credit line to the material. If material is not included in the article's Creative Commons licence and your intended use is not permitted by statutory regulation or exceeds the permitted use, you will need to obtain permission directly from the copyright holder. To view a copy of this licence, visit http://creativecommons.org/licenses/by/4.0/.

\section{References}

1. GallRiks Annual Report (2018) https://www.ucr.uu.se/gallriks/ fou/arsrapporter

2. Moller M et al (2014) Natural course vs interventions to clear common bile duct stones: data from the Swedish Registry for Gallstone Surgery and Endoscopic Retrograde Cholangiopancreatography (GallRiks). JAMA Surg 149(10):1008-1013

3. Boerma D, Schwartz MP (2006) Gallstone disease. Management of common bile-duct stones and associated gallbladder stones: surgical aspects. Best Pract Res Clin Gastroenterol 20(6):1103-1116

4. Caddy GR, Tham TC (2006) Gallstone disease: symptoms, diagnosis and endoscopic management of common bile duct stones. Best Pract Res Clin Gastroenterol 20(6):1085-1101

5. Grubnik VV et al (2012) Laparoscopic common bile duct exploration versus open surgery: comparative prospective randomized trial. Surg Endosc 26(8):2165-2171

6. Paganini AM et al (2007) Thirteen years' experience with laparoscopic transcystic common bile duct exploration for stones. Effectiveness and long-term results. Surg Endosc 21(1):34-40

7. Stromberg C, Nilsson M, Leijonmarck CE (2008) Stone clearance and risk factors for failure in laparoscopic transcystic exploration of the common bile duct. Surg Endosc 22(5):1194-1199

8. Zhang WJ et al (2015) Treatment of gallbladder stone with common bile duct stones in the laparoscopic era. BMC Surg 15:7

9. Enochsson L et al (2004) Intraoperative endoscopic retrograde cholangiopancreatography (ERCP) to remove common bile duct stones during routine laparoscopic cholecystectomy does not prolong hospitalization: a 2-year experience. Surg Endosc 18(3):367-371

10. Noel R et al (2013) A 10-year study of rendezvous intraoperative endoscopic retrograde cholangiography during cholecystectomy and the risk of post-ERCP pancreatitis. Surg Endosc 27(7):2498-2503 
11. Swahn F et al (2013) Rendezvous cannulation technique reduces post-ERCP pancreatitis: a prospective nationwide study of 12,718 ERCP procedures. Am J Gastroenterol 108(4):552-559

12. Swahn F et al (2013) Endoscopic retrograde cholangiopancreatography with rendezvous cannulation reduces pancreatic injury. World J Gastroenterol 19(36):6026-6034

13. Enochsson L et al (2015) GallRiks 10 years. Quality registry for gallstone surgery have improved health care. Lakartidningen 112:10

14. Elmunzer BJ et al (2017) The impact of cholecystectomy after endoscopic sphincterotomy for complicated gallstone disease. Am J Gastroenterol 112(10):1596-1602

15. Huang L et al (2015) The rendezvous technique for common bile duct stones: a meta-analysis. Surg Laparosc Endosc Percutan Tech 25(6):462-470

16. La Greca G et al (2009) Simultaneous laparoendoscopic rendezvous for the treatment of cholecystocholedocholithiasis. Surg Endosc 24(4):769-780

17. Lella F et al (2006) Use of the laparoscopic-endoscopic approach, the so-called "rendezvous" technique, in cholecystocholedocholithiasis: a valid method in cases with patient-related risk factors for post-ERCP pancreatitis. Surg Endosc 20(3):419-423

18. Diehl AK (2000) Cholelithiasis and the insulin resistance syndrome. Hepatology 31(2):528-530

19. Portincasa P, Moschetta A, Palasciano G (2006) Cholesterol gallstone disease. Lancet 368(9531):230-239

20. Chavez-Tapia NC et al (2012) Association between cholecystectomy for gallstone disease and risk factors for cardiovascular disease. Ann Hepatol 11(1):85-89

21. Shabanzadeh DM et al (2017) Screen-detected gallstone disease and cardiovascular disease. Eur J Epidemiol 32(6):501-510

22. Loureiro ER et al (2011) Laparoscopic cholecystectomy in 960 elderly patients. Rev Col Bras Cir 38(3):155-160

23. Andriulli A et al (2007) Incidence rates of post-ERCP complications: a systematic survey of prospective studies. Am J Gastroenterol 102(8):1781-1788

24. Painter NP, Kumar PA, Arora H (2014) Acute pulmonary embolism during an endoscopic retrograde cholangiopancreatography. Ann Card Anaesth 17(2):145-147
25. Stein PD, Matta F, Sabra MJ (2014) Pulmonary embolism and deep venous thrombosis following laparoscopic cholecystectomy. Clin Appl Thromb Hemost 20(3):233-237

26. Enochsson L et al (2013) The Swedish Registry of Gallstone Surgery and Endoscopic Retrograde Cholangiopancreatography (GallRiks): a nationwide registry for quality assurance of gallstone surgery. JAMA Surg 148(5):471-478

27. Rystedt J, Montgomery A, Persson G (2014) Completeness and correctness of cholecystectomy data in a national register-GallRiks. Scand J Surg 103(4):237-244

28. Roth GA et al (2017) Global, regional, and National Burden of Cardiovascular Diseases for 10 causes, 1990 to 2015. J Am Coll Cardiol 70(1):1-25

29. Cai JS, Qiang S, Bao-Bing Y (2017) Advances of recurrent risk factors and management of choledocholithiasis. Scand J Gastroenterol 52(1):34-43

30. Nassar Y, Richter S (2019) Management of complicated gallstones in the elderly: comparing surgical and non-surgical treatment options. Gastroenterol Rep (Oxf) 7(3):205-211

31. Scollay JM et al (2011) Mortality associated with the treatment of gallstone disease: a 10-year contemporary national experience. World J Surg 35(3):643-647

32. Mayhew D, Mendonca V, Murthy BVS (2019) A review of ASA physical status - historical perspectives and modern developments. Anaesthesia 74(3):373-379

33. Hallerback B, Enochsson L (2020) A prospective nationwide study on the impact of the level of sedation on cannulation success and complications of endoscopic retrograde cholangiopancreatography. Ann Gastroenterol 33(3):299-304

34. Syren EL et al (2019) Postoperative rendezvous endoscopic retrograde cholangiopancreaticography as an option in the management of choledocholithiasis. Surg Endosc. https://doi.org/10.1007/ s00464-019-07272-1

35. Loozen CS et al (2017) Early cholecystectomy for acute cholecystitis in the elderly population: a systematic review and metaanalysis. Dig Surg 34(5):371-379

Publisher's Note Springer Nature remains neutral with regard to jurisdictional claims in published maps and institutional affiliations. 